\title{
Derivation of a Suitable Finite Test Suite for Customized Probabilistic Systems ${ }^{\star}$
}

\author{
Luis F. Llana-Díaz, Manuel Núñez, and Ismael Rodríguez \\ Dept. Sistemas Informáticos y Programación \\ Universidad Complutense de Madrid, 28040 Madrid, Spain \\ \{llana, mn, isrodrig\}@sip.ucm.es
}

\begin{abstract}
In order to check the conformance of an IUT (implementation under test) with respect to a specification, it is not feasible, in general, to test the whole set of IUT available behaviors. In some situations, testing the behavior of the IUT assuming that it is stimulated by a given usage model is more appropriate. Specifically, if we consider that specifications and usage models are defined in probabilistic terms, then by applying a finite set of tests to the IUT we can compute a relevant metric: An upper bound of the probability that a user following the usage model finds an error in the IUT. We also present a method to find an optimal (with respect to the number of inputs) set of tests that minimizes that upper bound.
\end{abstract}

\section{Introduction}

In order to test the behavior of an IUT (implementation under test) sometimes it is preferable to check only some functionalities that are specially relevant or critical. In this line, we can consider that the IUT is analyzed in the context of a specific usage model or, more generally, in terms of its interaction with a (probably abstract) user that represents some manners to interact with the IUT. Let us note that if only the functional behavior of systems is considered (that is, we just check what must or must not be done), then this kind of user-customized approach consists in testing a subset of the behaviors defined by the specification. However, if other kinds of features are taken into account then this approach might provide some interesting possibilities. In particular, if specifications and user models are defined in probabilistic terms then we can calculate a measure that cannot be computed otherwise: After a finite set of tests is applied to the IUT, we can calculate a measure of the probability that a user behaving according to the user model finds a wrong behavior in the IUT. That is, after a finite subset of the infinite set of relevant behaviors is analyzed, we will be provided with a global measure of correctness of the IUT.

We can do it as follows. First, we choose some tests that exercise some behaviors concerned by the user model. Then, we apply the tests to the IUT to check

\footnotetext{
* Research partially supported by the Spanish MCYT project TIC2003-07848-C02-01, the Junta de Castilla-La Mancha project PAC-03-001, and the Marie Curie project MRTN-CT-2003-505121/TAROT.
} 
whether the behaviors exercised by these tests are correct with respect to the specification. Basically, tests induce some stimuli (sequences of inputs) and we observe the response (sequences of outputs) produced by the IUT. We cannot observe if the IUT produces $a$ with probability 0.5 ; what we can observe is the fact that $a$ is produced or not, but not the value 0.5 . In fact, our process to assess the IUT will be probabilistic. For each specific behavior case (i.e., sequence of inputs) analyzed by tests, we apply a hypothesis contrast to check whether we can claim, for a given level of confidence $\alpha$, that the answers (i.e., sequences of outputs) produced by the IUT behave as required by the specification. For instance, if the specification says that $a$ and $b$ are produced with 0.5 probability each, then the confidence will be high if they are observed 507 and 493 times, respectively. However, if they are produced 614 and 386 times then the confidence will be lower.

Let us suppose that, after a suitable hypothesis contrast is applied, a given IUT behavior case is validated with confidence $\alpha$. Then we can assume, with that confidence, that the probability of each IUT response (in that behavior case) is actually defined as in the specification. Under this confidence, we can calculate the probability that a sequence of inputs/outputs whose behavior was validated is produced during the interaction of the IUT and the user model. By using this information we will calculate our correctness metric: An upper bound of the probability that a wrong probabilistic behavior is observed when the user model and the IUT interact. In the worst case all behaviors that have not been either validated or observed are wrong. Hence, after a finite test suite is applied, we can compute the probability of taking a non-validated behavior, which is incorrect in the worst case (with confidence $\alpha$ ).

Let us note that this measure cannot be computed in other testing frameworks. In particular, if the probabilistic behavior of systems is not considered (or it is, but user models are not regarded), then after a finite test suite is applied the coverage of all relevant cases is null in general: Among an infinite set of behavior cases to be analyzed, an infinitesimal part of them are tested. Thus, without any additional assumptions, nothing about the correctness of this IUT can be claimed. Though the capability of a finite test suite to detect errors in an IUT can be assessed, it is done either in heuristic terms 8 102 or by adding some hypotheses about the IUT behavior that allow to reduce the number of cases to be tested [111211718]. On the contrary, our metric provides a (probabilistic) non-heuristic correctness measure without reducing the testing space.

In this paper we continue a previous work [12. In that approach, the behavior of the composition of the IUT and the user model is probabilistically compared to the behavior of the composition of the specification and the user model. A single hypothesis contrast is used to compare the former (denoted by means of a single random variable) and the latter (represented by a sample denoting all observations). Though this approach is simple and elegant, it has a drawback: Even if we assume that user models are correct by definition, their probabilistic behavior may be a source of sampling noise. For instance, let us suppose that the user model chooses between $a$ and $b$ with equal probability. Then, in both 
cases, the specification answers $c$ and $d$, again with equal probabilities. Though it is not very probable, the interaction of the user model and a correct IUT could produce $a / c 100$ times and $b / c 100$ times. In this case, we would (wrongly) deduce that the IUT is incorrect, because the specified probability of both $a / c$ and $b / c$ is $0.5 \cdot 0.5=0.25 \not \frac{100}{200}$. Similarly, $a / c$ and $a / d$ could be produced 100 times each, which again is not accepted. However, the latter sample is rejected due to a (rare) behavior of the user model. On the contrary, in this paper we will apply a hypothesis contrast for each behavior case (i.e., sequence of inputs). Each hypothesis contrast application checks whether responses (i.e., sequences of outputs) are properly given for the considered sequence of inputs. In the previous example, the correctness of the IUT when $a$ or $b$ are produced is independently checked. Hence, the sample cannot be ruined by a rare behavior of the user model (of course, it can still be ruined by a rare behavior of a correct IUT). Besides, in this paper we will use the metric of error probability of the IUT to find optimal sets of tests. Let us note that our metric does not only provide a correctness measure, but it also can be used to guide the testing process: If we compute that passing a given test suite $\Omega_{1}$ would provide an error measure 0.3 , while passing another suite $\Omega_{2}$ would provide an error measure 0.2 , then the suite $\Omega_{2}$ is preferable.

In terms of other related work, there is significant work on testing preorders and equivalences for probabilistic processes 41619|53201413. Most of these proposals follow the de Nicola and Hennessy's style [6], that is, two processes are equivalent if the application of any test belonging to a given set returns the same result. Instead, we are interested in checking whether an implementation conforms to a specification. In particular, our relations are similar to the ones introduced in [2115].

The rest of the paper is structured as follows. In the next section we present some basic notions; in Section 3 we introduce tests and we define the interaction between machines and users; in Section 4 we present the relations that allow to relate specifications and implementations; next, in Section 5 we describe our method to calculate an upper bound of the error probability of an IUT after it is tested, and we use this notion to find optimal test suites; finally, in Section 6 we present our conclusions and some lines of future work.

\section{Basic Notions}

First, we introduce some statistics notions. An event is any reaction we can detect from a system or environment; a random variable is a function associating each event with its probability.

Definition 1. Let $\mathcal{A}$ be a set of events and $\xi: \mathcal{A} \rightarrow[0,1]$ be a function such that $\sum_{\alpha \in \mathcal{A}} \xi(\alpha)=1$. We say that $\xi$ is a random variable for the set of events $\mathcal{A}$.

If we observe that the event $\alpha \in \mathcal{A}$ is produced by a random source whose probabilistic behavior is given by $\xi$ then we say that $\alpha$ has been generated by $\xi$. We extend this notion to sequences of events as expected: If we observe that the sequence of events $H=\left\langle\alpha_{1}, \ldots, \alpha_{n}\right\rangle$ is consecutively produced by a random 
source whose probabilistic behavior is given by $\xi$ then we say that $H$ has been generated by $\xi$ or that $H$ is a sample of $\xi$.

Given the random variable $\xi$ and a sequence of events $H$, we denote the confidence that $H$ is generated by $\xi$ by $\gamma(\xi, H)$.

This definition introduces a simple version of discrete random variable where all the events are independent. The actual definition of a random variable is more complex but it is pointless to use its generality in our setting. In the previous definition, the application of a suitable hypothesis contrast is abstracted by the function $\gamma$. We have that $\gamma(\xi, H)$ takes a value in $[0,1]$. Intuitively, a sample will be rejected if the probability of observing that sample from a given random variable is low. Due to lack of space we do not present here an actual definition of the function $\gamma$. An interested reader can find it in 12 . It is worth to point out that the results of this paper do not depend on the formulation of $\gamma$, being possible to abstract the actual definition.

Next we present the formalism we will use to define specifications and implementations. A probabilistic finite state machine is a finite state machine where each transition is equipped with a probability. Thus, a transition $s \stackrel{i / o}{\longrightarrow} s^{\prime}$ denotes that, if the machine is in state $s$ and the input $i$ is received then, with probability $p$, it moves to the state $s^{\prime}$ and produces the output $o$. We will assume that the environment stimulates the machine with a single input at any time. Thus, given $s$ and $i$, the addition of all values $p$ such that there exist $o$, $s^{\prime}$ with $s \stackrel{i / o}{\longrightarrow} p s^{\prime}$ must be equal to 1 . In contrast, there is no requirement binding the probabilities departing from the same state and receiving different inputs because each one describes (part of) a different probabilistic choice of the machine. In other words, we consider a reactive interpretation of probabilities (see [716]).

Definition 2. A Probabilistic Finite State Machine, in short PFSM, is a tuple $M=\left(S, I, O, \delta, s_{0}\right)$ where

$-S$ is the finite set of states and $s_{0} \in S$ is the initial state.

- $I$ and $O$, with $I \cap O=\varnothing$, denote the sets of input and output actions, respectively.

$-\delta \subseteq S \times I \times O \times(0,1] \times S$ is the set of transitions. We will write $s \stackrel{i / o}{\longrightarrow} s^{\prime}$ to denote $\left(s, i, o, p, s^{\prime}\right) \in \delta$.

Transitions and states fulfill the following additional conditions:

- For all $s \in S$ and $i \in I$, the probabilities associated with outgoing transitions add up to 1 , that is, $\sum\left\{p \mid \exists o \in O, s^{\prime} \in S: s \stackrel{i / o}{\longrightarrow} p s^{\prime}\right\}=1$.

- PFSMs are free of non-observable non-determinism, that is, if we have two transitions $s \stackrel{i / o}{\longrightarrow} p_{1} s_{1}$ and $s \stackrel{i / o}{\longrightarrow} p_{2} s_{2}$ then $p_{1}=p_{2}$ and $s_{1}=s_{2}$.

- In addition, we will assume that implementations are input-enabled, that is, for all state $s$ and input $i$ there exist $o, p, s^{\prime}$ such that $s \stackrel{i / o}{\longrightarrow} p s^{\prime}$. 
Although PFSMs will be used to define specifications and implementations, a different formalism will be used to define user models. Specifically, we will use probabilistic labeled transition systems. A user model represents the external environment of a system. User models actively produce inputs that stimulate the system, while passively receive outputs produced by the system as a response. The states of a user model are split into two categories: Input states and output states. In input states, all outgoing transitions denote a different input action. Since inputs are probabilistically chosen by user models, any input transition is endowed with a probability. In particular, $s \stackrel{i}{\longrightarrow} p s^{\prime}$ denotes that, with probability $p$, in the input state $s$, the input $i$ is produced and the state is moved to $s^{\prime}$. Given an input state $s$, the addition of all probabilities $p$ such that there exists $i, s^{\prime}$ with $s \stackrel{i}{\longrightarrow} p s^{\prime}$ must be lower than or equal to 1 . If it is lower then we will consider that the remainder up to 1 implicitly denotes the probability that the interaction with the system finishes at the current state. Regarding output states, all transitions departing from an output state are labeled by a different output action. However, output transitions do not have any probability value (let us remind that outputs are chosen by the system). Input and output states will strictly alternate, that is, for any input state $s$, with $s \stackrel{i}{\longrightarrow} p s^{\prime}, s^{\prime}$ is an output state, and for any output state $s$, with $s \stackrel{o}{\longrightarrow} s^{\prime}, s^{\prime}$ is an input state.

Definition 3. A probabilistic labeled transition system, in short PLTS, is a tuple $U=\left(S_{I}, S_{O}, I, O, \delta, s_{0}\right)$ where

- $S_{I}$ and $S_{O}$, with $S_{I} \cap S_{O}=\varnothing$, are the finite sets of input and output states, respectively. $s_{0} \in S_{I}$ is the initial state.

- $I$ and $O$, with $I \cap O=\varnothing$, are the sets of input and output actions, respectively.

$-\delta \subseteq\left(S_{I} \times I \times(0,1] \times S_{O}\right) \cup\left(S_{O} \times O \times S_{I}\right)$ is the transition relation. We will write $s \stackrel{i}{\longrightarrow} p s^{\prime}$ to denote $\left(s, i, p, s^{\prime}\right) \in S_{I} \times I \times(0,1] \times S_{O}$ and $s \stackrel{o}{\longrightarrow} s^{\prime}$ to denote $\left(s, o, s^{\prime}\right) \in S_{O} \times O \times S_{I}$.

Transitions and states fulfill the following additional conditions:

- For all input states $s \in S_{I}$ and input actions $i \in I$ there exists at most one outgoing transition from $s:\left|\left\{s \stackrel{i}{\longrightarrow} p s^{\prime} \mid \exists p \in(0,1], s^{\prime} \in S_{O}\right\}\right| \leq 1$.

- For all output states $s \in S_{O}$ and output actions $o \in O$ there exists exactly one outgoing transition labeled with $o:\left|\left\{s \stackrel{o}{\longrightarrow} s^{\prime} \mid \exists s^{\prime} \in S_{I}\right\}\right|=1$.

- For all input state $s \in S_{I}$ the addition of the probabilities associated with the outgoing transitions is lower than or equal to 1 , that is, $\operatorname{cont}(s)=$ $\sum\left\{p \mid \exists s^{\prime} \in S_{O}: s \stackrel{i}{\longrightarrow} p s^{\prime}\right\} \leq 1$. So, the probability of stopping at that state $s$ is $\operatorname{stop}(s)=1-\operatorname{cont}(s)$.

By iteratively executing transitions, both PFSMs and PLTSs can produce sequences of inputs and outputs. The probabilities of these sequences are given by the probabilities of the transitions. Next we introduce some trace notions.

Definition 4. A probability trace $\pi$ is a finite sequence of probabilities, that is, a possibly empty sequence $\left\langle p_{1}, p_{2}, \ldots, p_{n}\right\rangle \in(0,1]^{*}$. The symbol $\epsilon$ denotes 
the empty probability trace. Let $\pi=\left\langle p_{1}, p_{2}, \ldots, p_{n}\right\rangle$ be a probability trace. We define its sef-product, denoted by $\prod \pi$, as $\prod_{1 \leq i \leq n} p_{i}$. Since $\prod_{a \in \varnothing}=1$, we have $\prod \epsilon=1$. Let $\pi=\left\langle p_{1}, p_{2}, \ldots, p_{n}\right\rangle$ and $\pi^{\prime}=\left\langle p_{1}^{\prime}, p_{2}^{\top}, \ldots, p_{m}^{\prime}\right\rangle$ be probability traces. Then, $\pi \cdot \pi^{\prime}$ denotes their concatenation that is, $\left\langle p_{1}, p_{2}, \ldots, p_{n}, p_{1}^{\prime}, p_{2}^{\prime}, \ldots, p_{m}^{\prime}\right\rangle$, while $\pi * \pi^{\prime}$ and $\pi / \pi^{\prime}$ denote their pairwise product and division respectively, that is, $\left\langle p_{1} * p_{1}^{\prime}, p_{2} * p_{2}^{\prime}, \ldots, p_{r} * p_{r}^{\prime}\right\rangle$ and $\left\langle p_{1} / p_{1}^{\prime}, p_{2} / p_{2}^{\prime}, \ldots, p_{r} / p_{r}^{\prime}\right\rangle$, where $r=\min (n, m)$.

A trace $\rho$ is a finite sequence of input/output actions $\left(i_{1} / o_{1}, i_{2} / o_{2}, \ldots, i_{n} / o_{n}\right)$. The symbol $\epsilon$ denotes the empty trace. Let $\rho$ and $\rho^{\prime}$ be traces. Then, $\rho \cdot \rho^{\prime}$ denotes their concatenation. A probabilistic trace is a pair $(\rho, \pi)$ where $\rho$ is a trace $\left(i_{1} / o_{1}, i_{2} / o_{2}, \ldots, i_{n} / o_{n}\right)$ and $\pi=\left\langle p_{1}, p_{2}, \ldots, p_{n}\right\rangle$ is a probability trace. If $\rho$ and $\pi$ are both empty then we have the empty probabilistic trace, written as $(\epsilon, \epsilon)$. Let $(\rho, \pi)$ and $\left(\rho^{\prime}, \pi^{\prime}\right)$ be probabilistic traces. Then, $(\rho, \pi) \cdot\left(\rho^{\prime}, \pi^{\prime}\right)$ denotes their concatenation, that is, $\left(\rho \cdot \rho^{\prime}, \pi \cdot \pi^{\prime}\right)$.

An input trace $\varrho$ is a finite sequence of input actions $\left(i_{1}, i_{2}, \ldots, i_{n}\right)$. We extend the previous notions of empty trace and concatenations to input traces in the expected way. If $\rho=\left(i_{1} / o_{1}, i_{2} / o_{2}, \ldots, i_{n} / o_{n}\right)$ then we denote by $\mathrm{i}(\rho)$ the input trace $\left(i_{1}, i_{2}, \ldots, i_{n}\right)$. A probabilistic input trace is a pair $(\varrho, \pi)$ where $\varrho$ is an input trace $\left(i_{1}, i_{2}, \ldots, i_{n}\right)$ and $\pi=\left\langle p_{1}, p_{2}, \ldots, p_{n}\right\rangle$. We also consider the concepts of concatenation and empty probabilistic input traces.

Next we define how to extract traces from PFSMs and PLTSs. First, we consider the reflexive and transitive closure of the transition relation, and we call it generalized transition. Then, probabilistic traces are constructed from generalized transitions by considering their sequences of actions and probabilities.

Definition 5. Let $M=\left(S, I, O, \delta, s_{0}\right)$ be a PFSM. We inductively define the generalized transitions of $M$ as follows:

- We have that $s \stackrel{\epsilon}{\Longrightarrow} s$ is a generalized transition of $M$ for all $s \in S$.

- If $s \stackrel{\rho}{\longrightarrow} s^{\prime}$ and $s^{\prime} \stackrel{i / o}{\longrightarrow} p s_{1}$ then $s \stackrel{\rho \cdot i / o}{\longrightarrow} \pi \cdot\langle p\rangle s_{1}$ is a generalized transition of $M$.

We say that $(\rho, \pi)$ is a probabilistic trace of $M$ if there exists $s \in S$ such that $s_{0} \stackrel{\rho}{\Longrightarrow} \pi$. In addition, we say that $\rho$ is a trace of $M$ and that $i(\rho)$ is an input trace of $M$. The sets $\operatorname{p} \operatorname{Tr}(M), \operatorname{tr}(M), i \operatorname{Tr}(M)$ denote the sets of probabilistic traces, traces, and input traces of $M$, respectively.

The previous notions can also be defined for PLTSs. In order to obtain sequences of paired inputs and outputs, traces begin and end at input states.

Definition 6. Let $U=\left(S_{I}, S_{O}, I, O, \delta, s_{0}\right)$ be a PLTS. We inductively define the generalized transitions of $U$ as follows:

- We have that $s \stackrel{\epsilon}{\Longrightarrow} s$ is a generalized transition of $U$ for all $s \in S_{I}$.

- If $s \in S_{I}, s \stackrel{\rho}{\longrightarrow} \pi s^{\prime}$, and $s^{\prime} \stackrel{i}{\longrightarrow} p s^{\prime \prime} \stackrel{o}{\longrightarrow} s_{1}$ then $s \stackrel{\rho \cdot i / o}{\longrightarrow} \pi \cdot\langle p\rangle s_{1}$ is a generalized transition of $U$. 
We say that $(\rho, \pi)$ is a probabilistic trace of $U$ if there exists $s \in S_{I}$ such that $s_{0} \stackrel{\rho}{\Longrightarrow} \pi$ s. In that case we will also say that $(\mathrm{i}(\rho), \pi)$ is a probabilistic input trace of $U$. In addition, we say that $\rho$ is a trace of $U$ and that $i(\rho)$ is an input trace of $U$. We define the probability of $U$ to stop after $\rho$, denoted by $\operatorname{stop}_{U}(\rho)$, as stop $(s)$. The sets $\operatorname{prr}(U), \operatorname{pi} \operatorname{Tr}(U), \operatorname{tr}(U)$ and $i \operatorname{Tr}(U)$ denote the set of probabilistic traces, traces, and input traces of $U$ respectively.

Next we identify PLTS that terminate, that is, such that all infinite traces have probability 0 .

Definition 7. Let $U$ be a PLTS. We say that $U$ is a terminating PLTS if for all $s$ such that there exists $\rho$ and $\pi$ with $s_{0} \stackrel{\rho}{\Longrightarrow} s$ we have that there exists $s^{\prime}, \rho^{\prime}, \pi^{\prime}$ such that $s \stackrel{\rho^{\prime}}{\Longrightarrow} \pi^{\prime} s^{\prime}$ and $\operatorname{stop}_{U}\left(\rho \cdot \rho^{\prime}\right)>0$.

Proposition 1. A PLTS $U$ is terminating iff $\sum_{(\rho, \pi) \in \operatorname{pTr}(U)}\left(\prod \pi\right) * \operatorname{stop}_{U}(\rho)=1$

As we will see, PLTS will be used to denote user models. In particular, any user model will be supposed to be a terminating PLTS.

\section{Tests and Composition of Machines}

In this section we define our tests as well as the interaction between the notions introduced in the previous section (PFSMs and PLTSs). As we said before, we will use PLTSs to define the behavior of the external environment of a system, that is, a user model. Moreover, PLTSs are also appropriate to define the tests we will apply to an IUT. Tests are PLTSs fulfilling some additional conditions. Basically, a test defines a finite sequence of inputs; we will use them to check a given secuence of inputs. Since tests consider a single sequence of inputs, each intermediate input state of the sequence contains a single outgoing transition labeled by the next input and probability 1 . Output states offer transitions with different outputs.

Definition 8. A test $T=\left(S_{I}, S_{O}, I, O, \delta, s_{0}\right)$ is a PLTS such that for all $s \in S_{I}$ there is at most one transition $s \stackrel{i}{\longrightarrow} p s^{\prime}$ (and if it exists then $p=1$ ), and for all $s \in S_{O}$ there is at most one next input state $s \stackrel{o}{\longrightarrow} s^{\prime}$ with a continuation, that is, $\left|\left\{s^{\prime \prime} \mid \exists i \in I, o \in O, s^{\prime \prime \prime} \in S_{O}, p \in(0,1]: s \stackrel{o}{\longrightarrow} s^{\prime \prime} \stackrel{i}{\longrightarrow} p s^{\prime \prime \prime}\right\}\right| \leq 1$.

Let us note that, contrarily to other frameworks, tests are not provided with diagnostic capabilities on their own. In other words, tests do not have fail/success states. Since our framework is probabilistic, the requirements defined by specifications are given in probabilistic terms. As we will see in the next section, deciding whether the IUT conforms to the specification will also be done in probabilistic terms. In particular, we will consider whether it is feasible that the IUT behaves as if it were defined as the specification indicates. We will check this fact by means of a suitable hypothesis contrast. 
Our testing methodology consists in testing the behavior of a system under the assumption that it is stimulated by a given user model. Thus, tests will be extracted from the behavior of the user model. Next we show how a test is constructed from a probabilistic trace of a user model. The input and output states of the test are identified with natural numbers. All the input states (but the first one) are also endowed with an output action. In order to distinguish between input and output states we decorate them with ${ }^{\bullet}$ and ${ }^{\star}$, respectively. Tests extracted from user model sequences fulfill an additional condition: All input states reached from a given output state (via different outputs) are connected with the same output state through the same input, up to the end of the sequence. A single test can process any answer to a given sequence of inputs, that is, it detects any sequence of outputs produced by the IUT as response.

Definition 9. Let $\varrho=\left(i_{1}, i_{2}, \ldots, i_{r}\right)$ be an input trace, $I$ be a set of input actions such that $\left\{i_{1}, \ldots i_{r}\right\} \subseteq I$, and $O$ be a set of output actions. We define the test associated to $\varrho$, assoc $(\varrho)$, as the test $\left(S_{I T}, S_{O T}, I, O, \delta_{T}, 0^{\bullet}\right)$, where

$-S_{I T}=\left\{0^{\bullet}, r^{\bullet}\right\} \cup\left\{(j, o)^{\bullet} \mid o \in O, 1 \leq j \leq r\right\}$ and $S_{O T}=\left\{j^{\star} \mid 1 \leq j \leq r\right\}$.

- For all $1 \leq j<r, o \in O:(j, o)^{\bullet} \stackrel{i_{j+1}}{\longrightarrow} 1(j+1)^{\star}, j^{\star} \stackrel{o}{\longrightarrow}(j, o)^{\bullet} \in \delta_{T}$. We also have $0^{\bullet} \stackrel{i_{1}}{\longrightarrow} 0^{\star}$.

Next we define the composition of a PFSM (denoting either a specification or an IUT) with a PLTS (denoting either a user model or a test) in terms of its behavior, that is, in terms of traces and probabilistic traces. The set of traces is easily computed as the intersection of the traces produced by both components. In order to define the set of probabilistic traces, the ones provided by both components are considered. For a given input/output pair $i / o$, the probability of producing $i$ will be taken from the corresponding transition of the PLTS, while the probability of producing $o$ as a response to $i$ will be given by a transition of the PFSM. Let us note that the states of a specification do not necessarily define outgoing transitions for all available inputs, that is, specifications are not necessarily input-enabled. So, a PFSM representing a specification could not provide a response for an input produced by a PLTS. Since the specification does not define any behavior in this case, we will assume that the PFSM is allowed to produce any behavior from this point on. The composition of a PLTS and a PFSM will be constructed to check whether the traces defined by the specification are correctly produced by the implementation. Hence, undefined behaviors will not be considered relevant and will not provide any trace to the composition of the PLTS and the PFSM. In order to appropriately represent the probabilities of the relevant traces, their probabilities will be normalized if undefined behaviors appear. We illustrate this process in the following example.

Example 1. Let us suppose that a user model can produce the inputs $i_{1}, i_{2}$, and $i_{3}$ with probabilities $\frac{1}{2}, \frac{1}{4}$ and $\frac{1}{4}$, respectively. At the same time, the corresponding specification provides outgoing transitions with inputs $i_{1}$ and $i_{2}$, but not with $i_{3}$. Since the specification does not define any reaction to $i_{3}$, the probabilities 
of taking inputs $i_{1}$ or $i_{2}$ in the composition of the specification and the user model are normalized to denote that $i_{3}$ is not considered. So, the probability of $i_{1}$ becomes $\frac{1 / 2}{3 / 4}=\frac{2}{3}$ while the probability of $i_{2}$ is $\frac{1 / 4}{3 / 4}=\frac{1}{3}$.

The next definition finds an appropriate normalization factor when these situations appear (in the previous example, this factor is $\frac{3}{4}$ ). Besides, we show how to recompute the probabilities of all traces in a PLTS when only sequences of inputs that are accepted by a given PLTS are considered. Finally, we consider the behavior of the composition of a PFSM and a PLTS. The set of traces of this composition is provided by the intersection of the set of traces of each machine. In order find the probabilistic traces we consider, on the one hand, the probabilistic traces of the PFSM and, on the other hand, the probabilistic traces of the PLTS normalized to this PFSM.

Definition 10. Let $M=\left(S_{M}, I, O, \delta_{M}, s_{0 M}\right)$ be a PFSM and let us consider a PLTS $U=\left(S_{I U}, S_{O U}, I, O, \delta_{U}, s_{0 U}\right)$ such that $s_{0 M} \stackrel{\rho}{\Longrightarrow} \pi_{1} s_{1}$ and $s_{0 U} \stackrel{\rho}{\Longrightarrow} \pi_{2} s_{2}$. We define:

- The sum of the probabilities of continuing together after $\rho$ as

$$
\operatorname{cont}_{M \| U}(\rho)=\sum\left\{p \mid \begin{array}{c}
\exists i \in I, o \in O, s_{2}^{\prime} \in S_{O U}, s_{1}^{\prime} \in S_{M}, r \in(0,1]: \\
s_{2} \stackrel{i}{\longrightarrow} p s_{2}^{\prime} \wedge s_{1} \stackrel{i / o}{\longrightarrow} s_{1}^{\prime}
\end{array}\right\}
$$

- The normalization factor of $M \| U$ after $\rho$ as the sum of the previous probability plus the probability of $U$ to stop after $\rho$, that is $\operatorname{norm}_{M \| U}(\rho)=$ $\operatorname{cont}_{M \| U}(\rho)+\operatorname{stop}_{U}(\rho)$.

We inductively define the probabilistic traces of $U$ normalized to $M$ as follows:

$-(\epsilon, \epsilon)$ is a normalized probabilistic trace.

- Let $(\rho, \pi)$ be a normalized probabilistic trace. Let us suppose that we have $s_{0 M} \stackrel{\rho}{\Longrightarrow} \pi_{1} s_{1}^{\prime} \stackrel{i / o}{\longrightarrow} p_{1} s_{1}$ and $s_{0 U} \stackrel{\rho}{\Longrightarrow} \pi_{2} s_{2}^{\prime} s_{2}^{\prime} \stackrel{i}{\longrightarrow} p_{2} s^{\prime \prime} \stackrel{o}{\longrightarrow} s_{2}$. Then, $(\rho \cdot i / o, \pi \cdot\langle p\rangle)$ is a normalized probabilistic trace, where $p$ is the product of $p_{1}$ and $p_{2}$ normalized with respect to the normalization factor of $M \| U$ after $\rho$, that is, $p=\frac{p_{1} \cdot p_{2}}{\operatorname{norm}_{M \| U}(\rho)}$.

Let $(\rho, \pi)$ be a normalized probabilistic trace where we have $s_{0 U} \stackrel{\rho}{\Longrightarrow} \pi^{\prime} s$ for some $\pi^{\prime}, s$. We say that $(i(\rho), \pi)$ is a normalized probabilistic input trace. In addition, we say that $\rho$ is a normalized trace and that $i(\rho)$ is a normalized input trace. We define the probability of $U$ to stop after $\rho$ normalized to $M$, denoted by $\operatorname{nstop}_{U, M}(\rho)$, as $\frac{\text { stop }_{(s)}}{\operatorname{norm}_{M \| U}(\rho)}$. The sets $\operatorname{npTr}_{M}(U), \operatorname{npi} \operatorname{Tr}(U, M), \operatorname{ntr}(U, M)$ and $\mathrm{ni} \operatorname{Tr}(U, M)$ denote the set of normalized probabilistic traces, normalized traces, and normalized input traces of $U$ to $M$ respectively.

The set of traces generated by the composition of $M$ and $U$, denoted by $\operatorname{tr}(M \| U)$, is defined as $\operatorname{tr}(M) \cap \operatorname{tr}(U)$. The set of probabilistic traces generated by the composition of $M$ and $U$, denoted by $\operatorname{prr}(M \| U)$, is defined as

$$
\left\{\left(\rho, \pi_{1} * \pi_{2}\right) \mid\left(\rho, \pi_{1}\right) \in \operatorname{pTr}(M) \wedge\left(\rho, \pi_{2}\right) \in \operatorname{npTr}_{M}(U)\right\}
$$


The set of input traces generated by the composition of $M$ and $U$, denoted by $i \operatorname{Tr}(M \| U)$, is defined as the set $\{i(\rho) \mid \rho \in \operatorname{tr}(M \| U)\}$.

Proposition 2. Let $M$ be a PFSM and let $U$ be a PLTS, then

$$
\operatorname{tr}(M \| U)=\{\rho \mid \exists p \in(0,1]:(\rho, p) \in \operatorname{pTr}(M \| U)\}
$$

Let us remark that the probabilistic behavior of the traces belonging to the composition of PFSMs and PLTSs is completely specified: The probabilities of inputs are provided by the PLTS while the probabilities of outputs are given by the PFSM. Since our method consists in testing the behavior of the IUT for some sequences of inputs, we will be interested in taking those traces that share a given sequence of inputs. Next we develop these ideas for sequences and sets of sequences.

Definition 11. Let $\operatorname{Tr}$ be a set of traces and $\varrho$ an input trace. We define the set of traces of $\operatorname{Tr}$ modulo $\varrho$, denoted by $\operatorname{tr}_{\varrho}(\operatorname{Tr})$, as the set $\{\rho \mid i(\rho)=\varrho, \rho \in \operatorname{Tr}\}$. If $M$ is a PFSM and $U$ is a PLTS, for the sake of clarity, we write $\operatorname{tr}_{\varrho}(M), \operatorname{tr}_{\varrho}(U)$, and $\operatorname{tr}_{\varrho}(M \| U)$ instead of $\operatorname{tr}_{\varrho}(\operatorname{tr}(M)), \operatorname{tr}_{\varrho}(\operatorname{tr}(U))$, and $\operatorname{tr}_{\varrho}(\operatorname{tr}(M \| U))$, respectively. Let $\operatorname{Tr}$ be a set of traces and $i T r$ a set of input traces. We define the set of traces of $\operatorname{Tr}$ modulo $i T r$, denoted by $\operatorname{prob}_{p T r}(i T r)$, as the set $\left\{\operatorname{tr}_{\varrho}(\operatorname{Tr}) \mid \varrho \in i \operatorname{Tr}\right\}$.

We will construct a random variable denoting the probability of each trace in the composition of a specification and a user. Unfortunately, taking the probability associated to each trace in the composition is not appropriate. In fact, the sum of the probabilities of all traces may be higher than 1 . This is because traces denote events such that some of them include others. For instance, if the event $(a / b, c / d)$ is produced then we know that $(a / b)$ is also produced. We solve this problem by taking into account a factor that is not explicitly considered in the traces: The choice of a user to stop in a state. In particular, the event representing that $(a / b, c / d)$ is produced and, afterwards immediately, the user finishes does not imply that $(a / b)$ is produced and then the user stops.

Proposition 3. Let $M$ be a PFSM and let $U$ be a terminating PLTS. We have

$$
\sum_{(\rho, \pi) \in \operatorname{pTr}(M \| U)}\left(\prod \pi\right) * \text { nstop }_{U, M}(\rho)=1
$$

By the previous result, we can use traces up to termination to construct a random variable denoting the probability of observe any trace in the composition of a specification and a user.

Definition 12. Let $M$ be a PFSM and let $U$ be PLTS. We define the traces random variable of the composition of $M$ and $U$ as the function $\xi_{M \| U}: \operatorname{pTr}(M \| U) \longrightarrow$ $(0,1]$ such that for all $(\rho, \pi) \in \operatorname{pTr}(M \| U)$ we have

$$
\xi_{M \| U}(\rho)=\left(\prod \pi\right) * \text { nstop }_{U, M}(\rho)
$$




\section{Probabilistic Relations}

In this section we introduce our probabilistic conformance relations. Following our user customized approach, they relate an IUT and a user model with a specification and the same user model. These three elements will be related if the probabilistic behavior shown by the IUT when stimulated by the user model appropriately follows the corresponding behavior of the specification. In particular, we will compare the probabilistic traces of the composition of the IUT and the user with those corresponding to the composition of the specification and the user. Let us remind that IUTs are input-enabled but specifications might not be so. So, the IUT could define probabilistic traces including sequences of inputs that are not defined in the specification. Since there are no specification requirements for them, these behaviors will be ignored by the relation. In order to do it, an appropriate subset of the traces of the composition of the IUT and the user must be taken. The probability of each trace belonging to this set will be recomputed by considering a suitable normalization. Later we will see another relation where, due to practical reasons, this requirement will be relaxed.

Definition 13. Let $S, I$ be PFSMs and $U$ be a PLTS. We define the set of probabilistic traces generated by the implementation $I$ and the user model $U$ modulo the specification $S$, denoted by $\operatorname{pr}(I \| U)_{S}$ as the set

$$
\left\{\left(\rho, \pi_{i} * \pi_{o}\right) \mid \mathrm{i}(\rho) \in \mathrm{i} \operatorname{Tr}(S) \wedge\left(\rho, \pi_{i}\right) \in \operatorname{npTr}_{S}(U) \wedge\left(\rho, \pi_{o}\right) \in \operatorname{pTr}(I)\right\}
$$

Let $S, I$ be PFSMs and $U$ be a PLTS. We say that $I$ conforms to $S$ with respect to $U$, denoted by $I \operatorname{conf}_{U} S$, if $\operatorname{pTr}(I \| U)_{S}=\operatorname{pTr}(S \| U)$.

The previous result provides a diagnostic by comparing the complete set of traces of the composition of the specification and the user with the full set of traces of the implementation and the user (up to the specification). We can also perform local comparisons: A local diagnostic is obtained by comparing only those traces that have a given sequence of inputs. Though we can compare these traces by comparing their corresponding probabilities, we will manipulate these probabilities before. In particular, we will divide the probability of each of them by the probability its sequence of inputs. These values will denote the probability of performing the sequence of outputs of the trace provided that the sequence of inputs is the considered one. Though this transformation is not needed to perform the current comparison, using these probabilities will be useful in further analyses.

Definition 14. Let $A$ be a set of probabilistic traces and $(\varrho, \pi)$ be a probabilistic input trace. We define the restriction $A$ to $(\varrho, \pi)$, denoted by $A \backslash(\varrho, \pi)$, as the set $\left\{\left(\rho, \pi^{\prime} / \pi\right) \mid\left(\rho, \pi^{\prime}\right) \in A \wedge \mathrm{i}(\rho)=\varrho\right\}$.

Definition 15. Let $S, I$ be PFSMs, $U$ be a PLTS, and $(\varrho, \pi) \in \operatorname{npiTr}(U, S)$ such that $\varrho \in i \operatorname{Tr}(S)$. We say that $I$ conforms to $S$ with respect to $U$ in the input trace $\varrho$, denoted by $I \operatorname{conf}_{U, \varrho} S$, if $\operatorname{pTr}(I \| U)_{S} \backslash(\varrho, \pi)=\operatorname{pTr}(S \| U) \backslash(\varrho, \pi)$. 
Next we relate our notions of conformance and conformance for a given sequence of inputs. If we have local conformance for all sequences of inputs, then the global conformance is met.

Proposition 4. Let $S, I$ be PFSMs, and $U$ and be a PLTS, then $I \operatorname{conf}_{U} S$ iff for any probabilistic input trace $(\varrho, \pi) \in \operatorname{piTr}(U)$ such that $\varrho \in i \operatorname{Tr}(S)$ we have $I \operatorname{conf}_{U, \varrho} S$.

Our tests are designed to check any input trace. The parallel composition of the test with the specification $S \| T$ performs traces that are not present in the parallel composition of the user and the specification $S \| U$. However, if we remove the probabilities associated to the input trace in the user model then the probability of the traces that are in both compositions is the same. Thus, if the implementation conforms the specification with respect to the test $T$ (i.e. $\left.I \operatorname{conf}_{T} S\right)$, then it also conforms the specification with respect to the user in the trace (i.e. $I \operatorname{conf}_{U, \varrho} S$ ).

Proposition 5. Let $S$ be a PFSM, $U$ and be a PLTS, $(\varrho, \pi) \in \operatorname{npiTr}(U, S)$ such that $\varrho \in i \operatorname{Tr}(S)$, and $T=\operatorname{assoc}(\varrho)$. Then

- For all $\rho \in \operatorname{tr}(S \| U) \cap \operatorname{tr}(S \| T)$ we have $\xi_{S \| T}(\rho) * \prod \pi=\xi_{S \| U}(\rho)$

- if $I \operatorname{conf}_{T} S$ then $I \operatorname{conf}_{U, \varrho} S$.

Although the previous relation properly defines our probabilistic requirements, it cannot be used in practice because we cannot read the probability attached to a transition in a black-box IUT. Let us note that even though a single observation does not provide valuable information about the probability of an IUT trace, an approximation to this value can be calculated by interacting a high number of times with the IUT and analyzing its reactions. In particular, we can compare the empirical behavior of the IUT with the ideal behavior defined by the specification and check whether it is feasible that the IUT would have behaved like this if, internally, it were defined conforming to the specification. Depending on the empirical observations, this feasibility may be different. The feasibility degree of a set of samples with respect to its ideal probabilistic behavior (defined by a random variable) will be provided by a suitable contrast hypothesis. We will rewrite the previous relation $I \operatorname{conf}_{T} S$ in these terms.

Definition 16. Let $M$ be a PFSM and $U$ be a PLTS. We say that a sequence $\left\langle\rho_{1}, \rho_{2}, \ldots, \rho_{n}\right\rangle$ is a trace sample of $M \| U$ if it is generated by $\xi_{M \| U}$.

Definition 17. Let $S$ be a PFSM and $H=\left\langle\rho_{1}, \rho_{2}, \ldots, \rho_{n}\right\rangle$ be a sequence of traces. $H_{S}$ denotes the sub-sequence $\left\langle\rho_{r 1}, \rho_{r 2}, \ldots, \rho_{r n}\right\rangle$ of $H$ that contains all the probabilistic traces whose input sequences can be produced by $S$, that is, $i\left(\rho_{r i}\right) \in i \operatorname{Tr}(S)$.

Let $S$ and $I$ be PFSMs, and $U$ be a PLTS. Let $\varrho \in i \operatorname{Tr}(S), T=\operatorname{assoc}(\varrho)$, and $H=\left\langle\rho_{1}, \rho_{2}, \ldots, \rho_{n}\right\rangle$ be a trace sample of $I \| T$, and $0 \leq \alpha \leq 1$. We write $S \operatorname{conf}_{H}^{\alpha} I$ if $\gamma\left(\xi_{S \| T}, H_{S}\right) \geq \alpha$. 


\section{Optimal Test Suites}

In this section we will focus on two aspects: How to find a suitable test suite and how to provide a metric that allows us to measure the quality of test suites. Test suites will we be chosen when they have a good value in that metric.

\subsection{Testing Quality Measurement}

Let us suppose that we have a test suite. We apply each test to validate a single trace. By iterating the process for each test, we can get a set of validated input traces. Since not all the traces are checked, we have to know how accurate is our judgment about the correctness of the specification. We will measure this accuracy in probabilistic terms. We assume that only the tested and validated input traces are correct and that all the others are incorrect. So, the probability of executing one of those untested traces gives us an upper bound of the probability that the user finds an error in the implementation. In order to compute this upper bound, we have to calculate the probability with which the user executes one of the validated traces. The complementary of that probability will be the upper bound we are looking for. In order to compute those probabilities we use the random variable $\xi_{S \| U}$. For any tested input trace $\varrho$, its probability is equal to the probability of the set of traces of $S \| U$ whose input traces are those of $\varrho$.

Definition 18. Let $S$ be a PFSM and $U$ be a PLTS. Then,

1. If $\varrho \in i \operatorname{Tr}(S \| U)$ then we denote by $\operatorname{prob}_{S \| U}(\varrho)$ the probability of the set of events $\operatorname{tr}_{\varrho}(\operatorname{tr}(S \| U))$ assigned by the random variable $\xi_{S \| U}$.

2. For any set $i T r \subseteq i \operatorname{Tr}(S \| U)$, we denote by $\operatorname{prob}_{S \| U}(i T r)$ the probability of the set of events $\operatorname{tr}_{i \operatorname{Tr}}(\operatorname{p} \operatorname{Tr}(S \| U))$ assigned by $\xi_{S \| U}$.

In the random variable $\xi_{S \| U}$ we consider only full execution of traces, i.e. until the user decides to stop. For that reason we have that all events are independent.

Proposition 6. Let $S$ be a PFSM and $U$ be a PLTS. If $\varrho \in i \operatorname{Tr}(S \| U)$ then

$$
\operatorname{prob}_{S \| U}(\varrho)=\sum\left\{\left(\prod \pi\right) * \operatorname{nstop}_{U, S}(\rho) \mid \mathbf{i}(\rho)=\varrho \wedge(\rho, \pi) \in \operatorname{pTr}(S \| U)\right\}
$$

For any set $i T r \subseteq i \operatorname{Tr}(S \| U), \operatorname{prob}_{S \| U}(i T r)=\sum\left\{\operatorname{prob}_{S \| U}(\varrho) \mid \varrho \in i \operatorname{Tr}\right\}$.

Next we show how to compute the aforementioned upper bound. The scenario is the following. We have applied tests corresponding to some input traces and we have obtained some samples. Then, we consider only those traces such that the corresponding sample passes the hypothesis contrast. The upper bound that the user finds a error is calculated by considering that the rest of input traces behaves incorrectly. So, we calculate the probability to execute one of the validated traces, that is $\operatorname{prob}_{S \| U}(i T r)$, being the complementary probability the bound we are looking for. Let us remark that the IUT does not appear in the expression $\operatorname{prob}_{S \| U}(i T r)$. The reason is that we have already tested the implementation in 
the input traces of the set $i T r$. Thus, we can assume that the implementation behaves for those traces as indicated by the specification. Besides, we cannot compute that probability from the implementation since it is a black box: We can only test it and take samples from it.

Definition 19. Let $S, I$ be PFSMs and $U$ be a PLTS.

- Let $\varrho$ be an input trace, $H$ be a sample of $I \|$ assoc $(\varrho)$, and $\alpha$ be a feasibility degree. We say that $\varrho$ is $(H, \alpha)$-tested if $I \operatorname{conf}_{H}^{\alpha} S$.

- Let $i T r$ be a set of input traces and $\mathcal{H}$ be the set of samples $\left\{H_{\varrho} \mid \varrho \in\right.$ $i \operatorname{Tr}, H_{\varrho}$ is a sample of $\left.I \| \operatorname{assoc}(\varrho)\right\}$. We say that $i \operatorname{Tr}$ is $(\mathcal{H}, \alpha)$-tested if $I$ conf $_{H_{\varrho}}^{\alpha} S$ for all $\varrho \in i T r$.

Let $i \operatorname{Tr} \subseteq i \operatorname{Tr}(S \| U)$ be a $(\mathcal{H}, \alpha)$-tested set of input traces for a set of samples $\mathcal{H}$ and a feasibility degree $\alpha$. Then, the upper bound of error probability of the user $U$ to find and error in $I$ with respect to the input trace set $i T r, \operatorname{ubErr}_{i T r}^{\mathcal{H}, \alpha}(I, U)$, is the probabity of executing a trace $\rho$ such that $i(\rho) \notin i \operatorname{Tr}$ :

$$
\operatorname{ubErr}_{i T r}^{\mathcal{H}, \alpha}(I, U)=1-\operatorname{prob}_{S \| U}(i T r)
$$

\subsection{Obtaining a Good Test Suite}

Now we give a criteria to choose the best test suite. This criteria will be equivalent to the $0 / 1$ knapsack problem. Due to its intrinsic complexity, good enough test suites will be obtained by applying one of the known suboptimal algorithms.

Since each test checks a single input trace, our test suite will try to minimize the upper bound introduced in Definition 19. So, to find a good test suite is equivalent to find an input trace set $i T r$ that maximizes $\operatorname{prob}_{S \| U}(i T r)$. This will be our first criterium to choose our test suite. Obviously, the set that maximizes that probability is the whole set of input traces, that is usually infinite. We need another criteria to limit the number of tests to be applied. It will consist in minimizing the size of tests. Since each tests consists in a sequence of $n$ pairs input/output, it sends and receives exactly $n$ input/output actions. Then, we consider $n$ as the size of the test.

Definition 20. Let $\varrho=\left(i_{1}, i_{2}, \ldots i_{n}\right)$ be an input trace. We say that the length of the test $T=\operatorname{assoc}(\varrho)$ is $n$ and we write length $(T)=n$. Let $i \operatorname{Tr}$ be a set of input traces. We define the length of the set $\mathcal{T}=\{\operatorname{assoc}(\varrho) \mid \varrho \in i T r\}$, denoted by length $(\mathcal{T})$, as $\sum\{$ length $(T) \mid T \in \mathcal{T}\}$

Let $S$ be a PFSM and $U$ be a PLTS. Let $n \in \mathbb{N}$ and $i T r \subseteq i \operatorname{Tr}(S \| U)$. We say that the set of tests $\mathcal{T}=\{\operatorname{assoc}(\varrho) \mid \varrho \in i T r\}$, with length $(\mathcal{T}) \leq n$, is $n$-optimum if there does not exist another set of traces $i \operatorname{Tr}^{\prime} \subseteq i \operatorname{Tr}(S \| U)$ and a set of tests $\mathcal{T}^{\prime}=\left\{\operatorname{assoc}(\varrho) \mid \varrho \in i \operatorname{Tr}^{\prime}\right\}$ with length $\left(\mathcal{T}^{\prime}\right) \leq n$ such that $\operatorname{prob}_{S \| U}\left(i T r^{\prime}\right)>\operatorname{prob}_{S \| U}(i T r)$.

Let us note that, since each trace is independent from the others, the problem to find an $n$-optimum test suite is equivalent to the $0 / 1$ knapsack problem: The 
total size of the knapsack is $n$; the elements are the input traces $\varrho=\left(i_{1}, \ldots, i_{r}\right) \in$ $i \operatorname{Tr}(S \| U)$ such that $r \leq n$; the cost of the trace $\varrho=\left(i_{1}, \ldots, i_{r}\right)$ is $r$; the value of a trace $\varrho$ is $\operatorname{prob}_{S \| U}(\varrho)$. Due to the intrinsic complexity of that problem, it is not feasible to find an $n$-optimum test suite. However, we can consider one of the suboptimal well-known algorithms to solve the problem (see for example [22]).

\subsection{Testing Methodology}

Finally, let us briefly sketch our testing methodology:

1. We fix $n$, the combined size of tests belonging to the suite, and $\alpha$, the feasibility degree to pass the hypotheses contrast.

2. We find a suboptimal test suite $\mathcal{T}$, corresponding to a set of input traces, for the size $n$.

3. We generate the trace sample $H_{T}$ for all test $T \in \mathcal{T}$.

4. We consider the set of input traces whose samples pass the hypotheses contrast with the required feasibility degree $\mathcal{H}=\left\{H_{T} \mid \exists T \in \mathcal{T}: I \operatorname{conf}_{H_{T}}^{\alpha} S\right\}$, $i \operatorname{Tr}=\left\{\varrho \mid \exists T \in \mathcal{T}: H_{T} \in \mathcal{H} \wedge T=\operatorname{assoc}(\varrho)\right\}$.

5. We calculate the probability of error $\operatorname{ubErr}_{i T r}^{\mathcal{H}, \alpha}(I, U)$.

\section{Conclusions and Future Work}

In this paper we have presented a formal methodology to test probabilistic systems that are stimulated according to a given user model. In particular, we compare the behavior of a specification when it is stimulated by a user model with the behavior of an IUT when it is stimulated by the same model. By taking into account the probabilities of systems we have that, after a finite test suite is applied to the IUT, we can measure, for a given confidence degree, an upper bound of the probability that a user behaving as the user model finds an error in the IUT. Though a previous work [12] introduces a first approach to compute this metric, this method lies in the idea of comparing a single random variable denoting all the behaviors in the composition of the specification and the user with a sample denoting the behavior of the IUT when the user stimulates it. On the contrary, in this paper we separately study the behavior of the IUT for each sequence of inputs. Hence, the frequency of sequences of inputs is not part of the sampled information. This approach requires to use a specific random variable for each sequence of inputs (instead of a single random variable for all traces), as well as separately validating each sample with respect to its corresponding random variable. We use the method to find, for a given number of input actions, a optimal finite test suite, that is, a suite such that if it is passed then the upper bound of error probability is lower that the value obtained with any other test suite of the same size.

As future work, we plan to compact the information collected by samples. Let us suppose that a sample $(a / x, b / y, c / z)$ is obtained. This implies that if the sequence of inputs $(a, b)$ would have been offered instead of $(a, b, c)$, then the sequence of outputs $(x, y)$ would have obtained. That is, if the sample 
$(a / x, b / y, c / z)$ is obtained then the sample $(a / x, b / y)$ is also obtained, as well as $(a / x)$. Hence, by considering all prefixes of a sample, the number of observations for some sequences of inputs increases. Since the precision of hypothesis contrasts is higher when the size of samples is higher, this approach would allow us to improve the precision of our probabilistic method.

\section{References}

1. B.S. Bosik and M.U. Uyar. Finite state machine based formal methods in protocol conformance testing. Computer Networks \& ISDN Systems, 22:7-33, 1991.

2. L. Bottaci and E.S. Mresa. Efficiency of mutation operators and selective mutation strategies: An empirical study. Software Testing, Verification and Reliability, 9:205232, 1999.

3. D. Cazorla, F. Cuartero, V. Valero, F.L. Pelayo, and J.J. Pardo. Algebraic theory of probabilistic and non-deterministic processes. Journal of Logic and Algebraic Programming, 55(1-2):57-103, 2003.

4. I. Christoff. Testing equivalences and fully abstract models for probabilistic processes. In CONCUR'90, LNCS 458, pages 126-140. Springer, 1990.

5. R. Cleaveland, Z. Dayar, S.A. Smolka, and S. Yuen. Testing preorders for probabilistic processes. Information and Computation, 154(2):93-148, 1999.

6. R. de Nicola and M.C.B. Hennessy. Testing equivalences for processes. Theoretical Computer Science, 34:83-133, 1984.

7. R. van Glabbeek, S.A. Smolka, and B. Steffen. Reactive, generative and stratified models of probabilistic processes. Information and Computation, 121(1):59-80, 1995.

8. R.G. Hamlet. Testing programs with the aid of a compiler. IEEE Transactions on Software Engineering, 3:279-290, 1977.

9. M. Hennessy. Algebraic Theory of Processes. MIT Press, 1988.

10. W.E. Howden. Weak mutation testing and completeness of test sets. IEEE Transactions on Software Engineering, 8:371-379, 1982.

11. D. Lee and M. Yannakakis. Principles and methods of testing finite state machines: A survey. Proceedings of the IEEE, 84(8):1090-1123, 1996.

12. L.F. Llana-Díaz, M. Núñez, and I. Rodríguez. Customized testing for probabilistic systems. In 18th Int. Conf. on Testing Communicating Systems, TestCom 2006, LNCS 3964, pages 87-102. Springer, 2006.

13. N. López, M. Núñez, and I. Rodríguez. Specification, testing and implementation relations for symbolic-probabilistic systems. Theoretical Computer Science, 353(13):228-248, 2006.

14. M. Núñez. Algebraic theory of probabilistic processes. Journal of Logic and Algebraic Programming, 56(1-2):117-177, 2003.

15. M. Núñez and I. Rodríguez. Encoding PAMR into (timed) EFSMs. In 22nd IFIP Conf. on Formal Techniques for Networked and Distributed Systems, FORTE 2002, LNCS 2529, pages 1-16. Springer, 2002.

16. M. Núñez and D. de Frutos. Testing semantics for probabilistic LOTOS. In Formal Description Techniques VIII, pages 365-380. Chapman \& Hall, 1995.

17. A. Petrenko. Fault model-driven test derivation from finite state models: Annotated bibliography. In 4th Summer School, MOVEP 2000, LNCS 2067, pages 196-205. Springer, 2001. 
18. I. Rodríguez, M.G. Merayo, and M. Núñez. A logic for assessing sets of heterogeneous testing hypotheses. In 18th Int. Conf. on Testing Communicating Systems, TestCom 2006, LNCS 3964, pages 39-54. Springer, 2006.

19. R. Segala. Testing probabilistic automata. In CONCUR'96, LNCS 1119, pages 299-314. Springer, 1996.

20. M. Stoelinga and F. Vaandrager. A testing scenario for probabilistic automata. In ICALP 2003, LNCS 2719, pages 464-477. Springer, 2003.

21. J. Tretmans. Test generation with inputs, outputs and repetitive quiescence. Software - Concepts and Tools, 17(3):103-120, 1996.

22. V. V. Vazirani. Approximation Algorithms. Springer-Verlag, 2001. 